archives-ouvertes

\title{
Contribution à la connaissance du paléoenvironnement de la vallée de la Deûle (Nord, France) : approche stratigraphique
}

Laurent Deschodt, Pierre-Gil Salvador

\section{To cite this version:}

Laurent Deschodt, Pierre-Gil Salvador. Contribution à la connaissance du paléoenvironnement de la vallée de la Deûle (Nord, France) : approche stratigraphique. Revue du Nord, Association Revue du Nord, 2003, 353 (5), pp.23. 10.3917/rdn.353.0023 . hal-02055011

\section{HAL Id: hal-02055011 https://hal-inrap.archives-ouvertes.fr/hal-02055011}

Submitted on 3 Mar 2019

HAL is a multi-disciplinary open access archive for the deposit and dissemination of scientific research documents, whether they are published or not. The documents may come from teaching and research institutions in France or abroad, or from public or private research centers.
L'archive ouverte pluridisciplinaire HAL, est destinée au dépôt et à la diffusion de documents scientifiques de niveau recherche, publiés ou non, émanant des établissements d'enseignement et de recherche français ou étrangers, des laboratoires publics ou privés. 


\section{Contribution à la connaissance du paléoenvironnement de la vallée de la Deûle (Nord, France) : approche stratigraphique}

Riches en ressources alimentaires, axes de communication privilégiés, zones refuge, les cours d'eau et les plaines alluviales qu'ils drainent ont engendré dans leur proximité une intense activité humaine, indissociable de leur évolution. L'approche géoarchéologique cherche à définir ces interrelations entre le cours d'eau et les sociétés du passé. Une des difficultés majeures de cette recherche réside dans la complexité et la variabilité du comportement des fleuves et des rivières. Les recherches entreprises depuis le $\mathrm{XIX}^{\mathrm{e}}$ siècle dans le domaine de l'hydrologie continentale et de la géomorphologie nous enseignent en effet qu'un cours d'eau n'est pas réductible à un simple linéaire, un canal évacuateur des eaux superficielles, mais que son fonctionnement est indissociable du bassin versant - versants et fond de vallée - dans lequel il s'inscrit. L'ensemble constitue un véritable « système fluvial » dont les éléments constitutifs interagissent de manière complexe ${ }^{1}$. On en soulignera le caractère évolutif, qu'illustre par exemple la mobilité spatiale et

\footnotetext{
*. - Laurent Deschodt, INRAP Nord-Picardie, 518 rue SaintFuscien, 80000 Amiens, courriel: 1deschodt@nordnet.fr; PierreGil SALVADOR, maître de conférences, Université Lille1, UFR de Géographie, UMR 8141 Laboratoire de géographie des milieux anthropisés, avenue Paul Langevin, 59655 Villeuneuve-d'Ascq CEDEX, courriel: pierre-gil.salvador@univ-lille1.fr. Nous remercions le laboratoire HALMA (Histoire, Archéologie, Littératures des Mondes Anciens), CNRS-UMR 8142, Université de Lille 3, qui a financé les analyses radiocarbones, et le Centrum voor isotoper onderzoek (Groningen University, Pays-Bas) qui les a réalisées.

1. - S. A. Schumm, The Fluvial System, J. Wiley and Sons, New York, 1977, 338 p.

2. - R. Blanchard, La Flandre. Étude géographique de la plaine flamande en France, Belgique et Hollande, Lille, 1906, 530 p., 76 fig., 2 pl. h.t.; A. Demangeon, La Picardie et les régions voisines - Artois, Cambrésis, Beauvaisis, Paris, 1905, 496 p.; P. PINCHEMEL, Les plaines $d u$ Nord-Ouest du Bassin Parisien et du Sud-Est du bassin de Londres et leurs bordures, Paris, 1954, 502 p., 49 fig., 22 pl. J. SommÉ, Les plaines du Nord de la France et leur bordure. Étude géomorphologique, Thèse d'État, Université Paris I, 1975, Paris, Champion, 1977, 2 vol. : 1-810, 185 fig.

3. - P. Antoine, « Modifications des systèmes fluviatiles à la transi-
}

temporelle des lits fluviaux. Les études paléoenvironnementales reconnaissent ainsi de nombreux cas de « métamorphose » de cours d'eau depuis l'époque tardiglaciaire.

Les travaux portant sur la nature et le fonctionnement des systèmes fluviaux du nord de la France (région Nord-Pas-de-Calais) se sont multipliés tout au long $\mathrm{du} \mathrm{XX}^{\mathrm{e}}$ siècle. Des informations ponctuelles (coupes stratigraphiques dans les vallées, sondages géologiques et autres observations de terrain) ont été publiées par les géologues et les géographes, ainsi que de grandes synthèses régionales, telles celles des géographes R. Blanchard, A. Demangeon, P. Pinchemel et J. Sommé 2 . Sans chercher l'exhaustivité, on complétera avec les travaux récents de P. Antoine ${ }^{3}$, centrés sur le bassin versant de la Somme et des études réalisées essentiellement lors d'opérations archéologiques dans la vallée de la Deûle ${ }^{4}$, de la Scarpe et de $1^{\prime}$ Escaut ${ }^{5}$. Toutes ces études contribuent à mettre en

\begin{abstract}
tion Pléniglaciaire-tardiglaciaire et à l'Holocène: l'exemple du bassin de la Somme (Nord de la France) ", Géographie physique et Quaternaire, 51, (1), 1997, p. 93-106.

4. - L. DeschoDT, Ébauche d'une cartographie au 1/10 000 des formations superficielles de Lille, Mémoire de DEA, septembre 1996, Université Lille 1, Les cahiers de préhistoire du Nord, 21-22, 1999, 208 p.; E. Teheux, L. Deschodt, Dourges, plate-forme multimodale. Rapport des évaluations paléolithiques à Hénin-Beaumont. Sites $n^{\circ} 62427025$ et $62427027 A H$, Rapport non publié, SRA, AFAN, 2000, $13 \mathrm{p}$.

5. - L. Deschodt, ZAC de Fresnes-sur-Escaut. Projet PARC. Campagne de pré-sondages archéologiques. Reconnaissance du contexte sédimentaire et évaluation du potentiel archéologique, Rapport non publié, AFAN, 2001, 61 p.; L. DESCHODT, «Une phase de sédimentation alluviale au Préboréal initial dans la vallée de l'Escaut (nord de la France) », Quaternaire, 13, (2), 2002, p. 149-152; A.-F. EMONTSPOHL, « Enregistrement pollinique du début du Weichsélien et de l'Holocène à Fampoux, vallée de la Scarpe (Nord, France) », Quaternaire, 5, (1), 1994, p. 35-40; N. RUCHARD, S. Lenaerts, A.-V. Munaut, «Étude palynologique de sédiments holocènes dans la plaine alluviale du Haut-Escaut (Nord, France) », Cahiers de la Préhistoire du Nord, 10, 1992, p. 118-130.
\end{abstract}




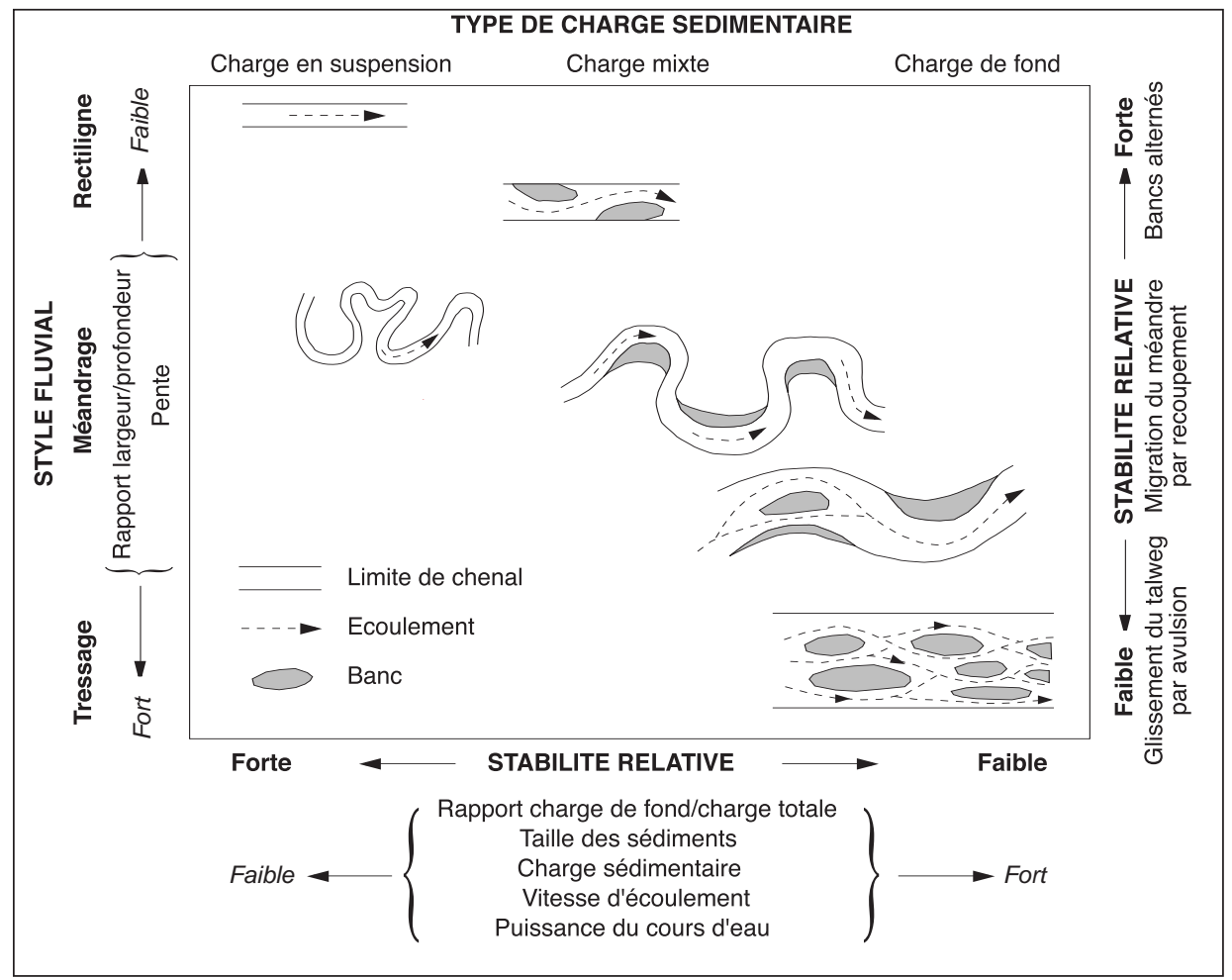

FIG. 1. - Diversité des styles fluviaux selon la nature de la charge sédimentaire. D'après Schumm et Meyer, 1979.

évidence l'originalité des rivières du nord de la France, marquées par un contexte paléogéographique et une dynamique naturelle spécifiques. L'action forte et déterminante de l'homme durant la période historique renforce le caractère tout à fait particulier de ces cours d'eau. L'objet de cet article est d'exposer les grands principes régissant la dynamique des cours d'eau, en insistant sur leur mobilité, puis de présenter les premiers résultats stratigraphiques obtenus dans la vallée de la Deûle.

\section{GÉNÉRALITÉS SUR LES ENREGISTREMENTS SÉDIMENTAIRES EN MILIEU FLUVIAL}

\subsection{Les rivières: une morphologie en équilibre instable}

\subsubsection{Un style fluvial dépendant de facteurs hydro- sédimentaires}

Les caractéristiques du bassin versant, conjuguées à l'action du climat et de l'homme, conditionnent les flux d'eau et de sédiments véhiculés par le réseau hydrographique. La réponse d'un cours d'eau à la combinaison des débits et de la charge sédimentaire, ainsi qu'aux différents facteurs de perturbation pouvant affecter localement l'écoulement (seuil rocheux, apports des affluents, rôle de la végétation...) est complexe. S'adaptant à ces multiples contraintes, les lits fluviaux offrent ainsi une gamme de formes très diversifiée. L'ajustement géomorphologique en plan détermine un style fluvial qui reflète des variations de niveaux d'énergie (puissance) et d'instabilité des lits (bancs, chenaux...) $)^{6}$. Le tressage définit par exemple des cours d'eau de haute énergie, associés à une grande mobilité du lit et suppose l'existence d'une charge solide abondante et de granulométrie plutôt grossière (fig. 1). Les rivières en tresses sont caractérisées par des chenaux larges, peu sinueux et de faible profondeur, enserrant des bancs sableux et caillouteux très mobiles. L'instabilité latérale est importante en raison de la faible cohésion des alluvions. Le style fluvial à méandres est quant à lui généralement associé à des milieux de moindre énergie et s'épanouit dans les sections aval des bassins versants aux reliefs moins

6. - J.-P. Bravard, F. Petit, Les cours d'eau, Paris, Armand Colin, 1997, 222 p. 
prononcés. Il se caractérise par la migration latérale d'un chenal unique et dissymétrique, défini par l'érosion de la berge externe et le dépôt des alluvions sur la berge interne (banc de convexité). Les trains de méandres, associant plusieurs sinuosités, migrent latéralement et en direction de l'aval. Des recoupements de boucles se produisent régulièrement et forment des bras morts parfois conservés à la surface de la plaine.

\subsubsection{Une sensibilité du système à l'origine des métamorphoses de la rivière}

Une variation des flux entrant dans le système fluvial, inféodée à des forçages de nature climatique et anthropique, peut alors engendrer des réajustements susceptibles de modifier profondément le comportement du cours d'eau et les paysages d'une même plaine alluviale au cours de son histoire ${ }^{7}$.

D’une façon générale et schématique, les périodes propices à la déstabilisation des versants déterminent de forts apports sédimentaires responsables de phases d'alluvionnement majeures dans les lits fluviaux. Le Néolithique moyen et récent, le premier âge du fer, une partie de l'Antiquité ( $\mathrm{I}^{\mathrm{er}}-\mathrm{III}^{\mathrm{e}}$ siècles ap. J.-C.), l'époque moderne sont, à l'échelle du domaine tempéré de l'Europe occidentale, des périodes de forte production sédimentaire. Ainsi par exemple, l'occurrence de la péjoration climatique du petit âge glaciaire (XIV ${ }^{\mathrm{e}}-\mathrm{XIX}{ }^{\mathrm{e}}$ siècles), conjuguée à une forte pression agro-pastorale, provoqua la propagation de nappes d'alluvions grossières depuis les torrents vers le fond des grandes vallées alpines ${ }^{8}$. Il s'ensuivit une évolution de nombreux cours d'eau d'un style fluvial à chenal unique en un style à tresses beaucoup plus instable, conforme aux nouvelles conditions de charge sédimentaire et de débit. Cette métamorphose fut très dommageable pour les activités humaines. L'élargissement important du lit mineur, jusqu'à plusieurs centaines de mètres, se fit au détriment des terres riveraines et son exhaussement, 2 à $3 \mathrm{~m}$ en moyenne sur l'Isère à l'aval de Grenoble, entraîna une dangereuse élévation des lignes d'eau facilitant les inondations et l'engravement des terres agricoles ${ }^{9}$.

À l'opposé, des périodes favorables à la stabilité des versants définissent une forte réduction des

7. - S. STARKEL, « The reflection of hydrologic changes in the fluvial environment of the temperate zone during the last 15000 years », dans Background to Palaeohydrology, K. J. Gregory, Chichester, 1983, p. 213-235.

8. - J.-P. BRAVARD, « La métamorphose des rivières des Alpes françaises à la fin du Moyen Âge et à l'époque moderne », Bulletin de la Société de Géographie de Liège, t. 25, 1989, p. 145-157.

9. - P.-G. SALVADOR, « L'évolution holocène de la plaine alluviale de l'Isère dans l'ombilic de Moirans (Isère, France) », Quaternaire, 12 , (1-2), 2001, p. 127-135.

10. - N. LANDON, L'évolution contemporaine du profil en long des apports solides. Cela contribue à l'incision des cours d'eau, qui pallient le déficit sédimentaire en puisant dans le stock alluvial que constitue le lit majeur. L'enfoncement contemporain des rivières du sud-est de la France, engendré par une raréfaction des flux sédimentaires, illustre ce type d'évolution. Ce déficit alluvionnaire s'explique par une combinaison d'impacts conjuguant un aménagement multi-fonctionnel des lits fluviaux (barrages, endiguements, extractions de granulats...) et une réduction naturelle de l'hydraulicité. L'incision atteint plusieurs mètres en moyenne, de 2 à $5 \mathrm{~m}$ sur la Drôme par exemple, sur des sections de plusieurs dizaines de kilomètres de longueur. Les conséquences économiques sont là encore très pénalisantes pour les communautés riveraines, avec de nombreux ouvrages d'art menacés par l'incision (digues, ponts...) et un abaissement des nappes phréatiques préjudiciable à l'exploitation agricole du lit majeur ${ }^{10}$.

\subsection{La plaine alluviale, un espace archivant l'his- toire de la rivière et des hommes}

Édifiée par le cours d'eau, la plaine alluviale constitue un espace composite qui juxtapose dans le temps toutes les formes engendrées par cette variabilité géomorphologique ${ }^{11}$. C'est souvent un milieu riche sur le plan archéologique, susceptible de fossiliser sur le long terme des sites d'habitat ou de simples structures témoignant des activités humaines passées. Mais là encore, les conditions de préservation des artéfacts dépendent de la paléo-dynamique fluviale ${ }^{12}$. Le potentiel archéologique des plaines de tressage est faible, eu égard au recouvrement et à la remobilisation des vestiges par d'épaisses nappes de galets. Ce style fluvial est d'ailleurs par nature peu propice - sans être rédhibitoire - à l'installation humaine, en raison de la forte activité morpho-dynamique qu'il engendre. Le potentiel archéologique est plus important dans les plaines à méandres, caractérisées par leur moindre dynamisme, en particulier lorsque la migration latérale des sinuosités est faible et favorable au phénomène d'accumulation verticale par les limons d'inondation. Les artéfacts se retrouveront préférentiellement dans la zone de dépôt constitué par le banc de convexité.

affluents du Rhône moyen. Constat régional et analyse d'un hydrosystème complexe, la Drôme, Thèse Géographie, Université Lyon 2, 1999, $560 \mathrm{p}$

11. - A. G. BRown, Alluvial Geoarchaeology. Floodplain Archaeology and Environmental Change, Cambridge Manuals in Archaeology, Cambridge University Press, 1997, 377 p.; J. LEwIN, «Floodplain Geomorphology », Progress in Physical Geography, 2, (3), 1978, p. 408-437.

12. - J.-P. BRavard, P.-G. SAlvador, « Éléments de géoarchéologie des plaines alluviales », dans Les sciences de la terre, Coll. « Pour l'Archéologue », Errance, 1999, p. 59-94. 
Le géologue ou le géomorphologue contribue à l'étude paléo-environnementale en interrogeant les archives sédimentaires stockées à plus ou moins long terme dans la plaine alluviale. L'analyse est conduite dans les trois dimensions de l'espace. Les coupes stratigraphiques et les sondages ponctuels permettent de reconnaître les unités sédimentaires et organiques, leur agencement, de même que l'analyse sédimentologique et micro-morphologique contribue à identifier la nature des sédiments (pétrographie, granulométrie...), les processus du dépôt (limons d'inondation, décantation dans un plan d'eau...) et son évolution (pédogenèse...). Les investigations menées à la surface de la plaine s'intéressent plus particulièrement aux variations de la topographie, souvent faibles en milieu alluvial. L'identification de niveaux de basses terrasses indique des phases successives d'alluvionnement et d'incision, de même que le repérage de paléochenaux peut conduire à l'identification de paléostyles fluviaux. Pour les périodes historiques, les traces des activités humaines sont parfois encore visibles à travers l'organisation des paysages actuels (paléo-réseau de fossés, parcellaire ancien...).

L'édification d'une plaine alluviale est ainsi le fruit d'une histoire complexe, d'autant plus difficile à reconstituer qu'elle est souvent influencée par l'incidence des activités humaines. L'approche géoarchéologique s'avère donc une démarche essentielle pour une meilleure compréhension des interrelations entre nature et sociétés du passé.

\section{SPÉCIFICITÉ DU RÉSEAU HYDROGRAPHIQUE DU NORD DE LA FRANCE}

\subsection{Un « haut » et un « bas » pays}

La région du Nord oppose schématiquement un bas pays, se développant en dessous de $80 \mathrm{~m}$ d'altitude, de la mer du Nord à l'Escaut, et un haut pays qui forme une écharpe de relief culminant autour de 180200 m d'altitude, depuis le Boulonnais jusqu'au Hainaut. Le haut pays constitue globalement une ligne de partage des eaux entre le réseau de la Somme, augmenté des fleuves côtiers comme l'Authie ou la Canche qui se jettent dans la Manche et l'ensemble des bassins de taille inégale qui drainent le bas pays, l'Aa, l'Yser, la Lys, l'Escaut et la Sambre, en dirigeant leurs eaux vers le nord (mer du Nord ou Belgique).

\subsection{De petites rivières dans de larges vallées}

Le réseau hydrographique du bas pays est formé essentiellement de petites rivières au débit limité, souvent artificialisé, coulant sur des pentes très faibles (inférieures à $1 \mathrm{~m} / \mathrm{km}$ dès que l'on quitte les versants du haut pays). Ces cours d'eau drainent cependant des vallées de largeur très variable, pour constituer parfois de vastes plaines humides (plaine de la Lys ou de la Scarpe par exemple). J. Sommé a déjà souligné cette distorsion dans le calibrage des vallées, engendrant une mauvaise adéquation entre l'hydrographie et les masses principales du relief ${ }^{13}$. Elle s'explique par un dispositif hérité de la tectonique, qui justifie également le tracé coudé de certains cours d'eau (Aa, Deûle, Escaut).

\subsection{Une forte sédimentation}

Un des faits majeurs de l'histoire paléogéographique de la région est le dépôt en masse de limons éoliens hérités des phases froides du Quaternaire. La couverture loessique a recouvert une partie de la région au Pléistocène supérieur. Elle est à l'origine d'un empâtement généralisé des reliefs. L'épaisseur cumulée des sédiments fluviatiles et loessiques peut dépasser $20 \mathrm{~m}$. L'Holocène apparaît comme une période de faible hydraulicité. La sédimentation fluviatile est modérée (quelques mètres d'épaisseur), avec des dépôts à dominante limoneuse et organique traduisant la faiblesse des écoulements. On se situe ainsi dans un contexte original où l'individualisation naturelle des cours d'eau holocènes, après l'ennoyage de la topographie par le loess, semble avoir été plutôt difficile. Comme le précise J. Sommé, l'anthropisation ancienne et intense du réseau hydrographique est donc probablement un facteur d'identification de ces cours d'eau, sous une forme artificialisée ${ }^{14}$.

\section{L'EXEMPLE DE LA DEÛLE}

\subsection{Une rivière caractéristique du bas-pays}

Affluent de la Lys et longue de $86 \mathrm{~km}$, la Deûle draine un bassin versant de $900 \mathrm{~km}^{2}$. Elle prend sa source à $100 \mathrm{~m}$ d'altitude sur les contreforts du BasArtois - il s'agit du cours de la Souchez - et rejoint rapidement les plaines du bas pays pour se jeter dans la Lys, à la hauteur de Deulémont (15 m d'altitude). Son régime hydrologique est de type océanique, caractérisé par des hautes eaux d'hiver et des basses 


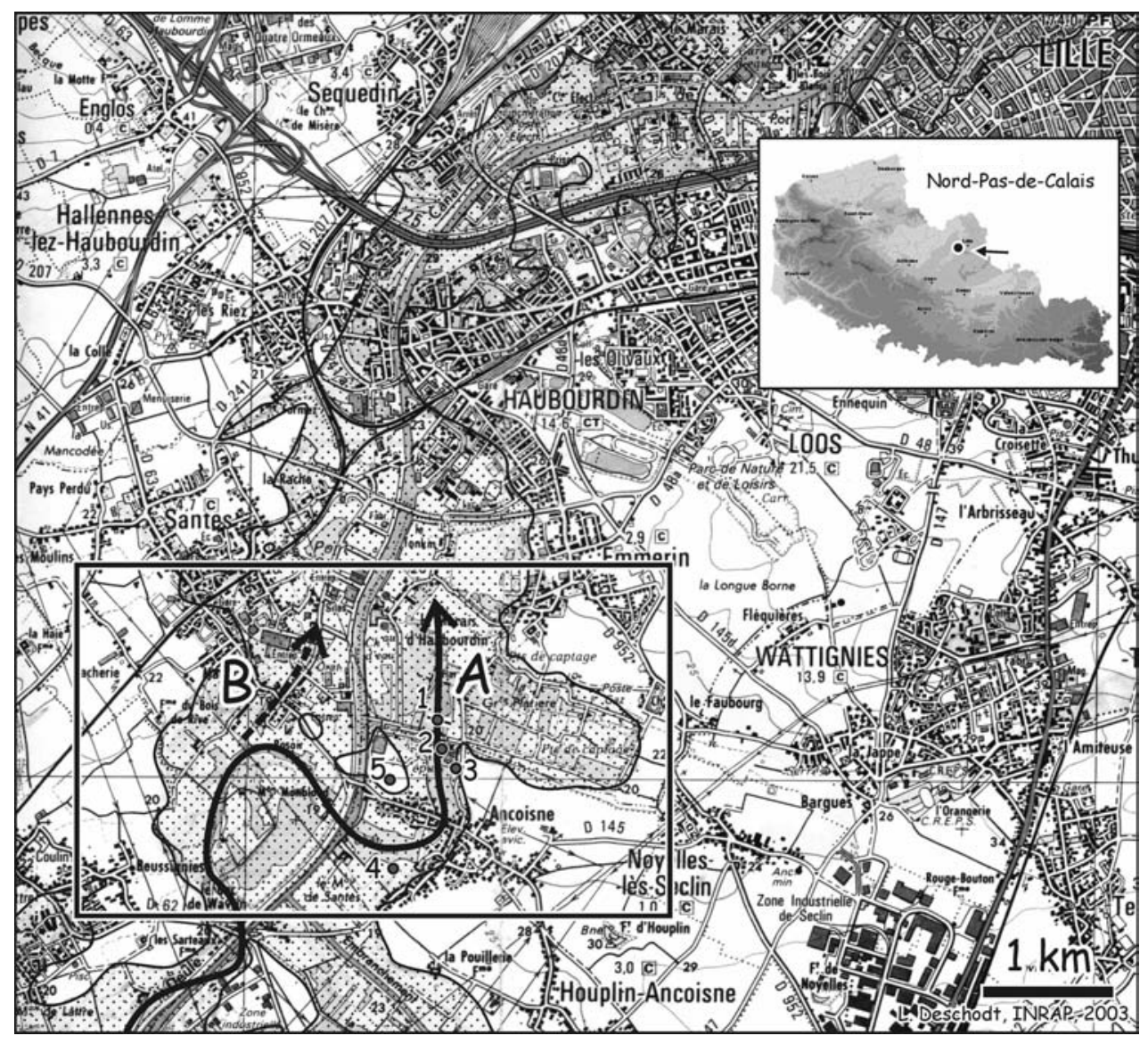

FIG. 2. - Localisation géographique du secteur d'Houplin-Ancoisne.

eaux estivales. Le débit moyen inter-annuel de la rivière à la station d'Haubourdin, juste à l'amont de Lille, est de $5 \mathrm{~m}^{3} / \mathrm{s}$ (période 1963-2003) ${ }^{15}$.

Dans le secteur d'étude (fig. 2), à HouplinAncoisne, la vallée s'étale sur une largeur de 2 à $3 \mathrm{~km}$, à une cote de 18-19 m NGF. Le fond de vallée sépare deux unités de reliefs modérés (points culminants vers 40 et $50 \mathrm{~m}$ ) mais distincts: le Pays de Weppes, à l'ouest, composé d'un substrat tertiaire argileux et sableux et d'un versant à couverture loessique épaisse (car protégé des vents dominants d'ouest) et le Mélantois, à l'est, dernier affleurement septentrional de craie, en forme de dôme, avec une couverture loes-

15. - Agence de l'eau, « Données sur l'eau », www.eau-artoispicardie.fr, 2002.

16. - A. DeRVILLE, « La maîtrise des eaux dans les Pays-Bas français avant Louis XIV », Les Pays Bas Français, $13^{e}$ Annales, 1988, p. 105123; A. DeRviLle, « Rivières et canaux du Nord/Pas-de-Calais aux époques médiévale et moderne », Revue du Nord, tome LXXII, n² 284 , 1990 , p. $5-22$

17. - N. Dessaux, S. Revillion, S. Delchambre, « Stratégies d'ex- sique modérée d'ordre métrique. Avant l'aménagement de la rivière à partir du XIII ${ }^{e}$ siècle $^{16}$, le fond de vallée se présentait sous la forme d'un lacis de chenaux et de tourbières. Les traces des anciens lits sont encore visibles dans la plaine alluviale, dont les marais furent exploités jusqu'à une époque récente ${ }^{17}$. En raison de la faiblesse des pentes, majoritairement inférieures à $1,5 \%$, la transition entre la plaine et les versants est insensible et rend incertain le tracé précis des limites ${ }^{18}$. L'observation détaillée des cartes IGN (fig. 3) permet de percevoir une microtopographie complexe: de larges méandres, des reliefs supérieurs à $20 \mathrm{~m}$ au cœur du fond de vallée, plusieurs lits mineurs...

ploitation et mutations du paysage dans la vallée de la haute Deûle (Nord) : l'exemple du marais de Seclin du XIII ${ }^{\mathrm{e}}$ au XIX ${ }^{\mathrm{e}}$ siècle », Revue du Nord, t. LXXX, n 326-327, 1998, p. 531-571.

18. - M. Franchomme, Conception et utilisation d'un SIG pour l'étude des données (paléo)environnementales et archéologiques appliqué au bassin versant de la Haute-Dê̂le, Mémoire de DEA, Université Lille 1, 2003, 118 p. 


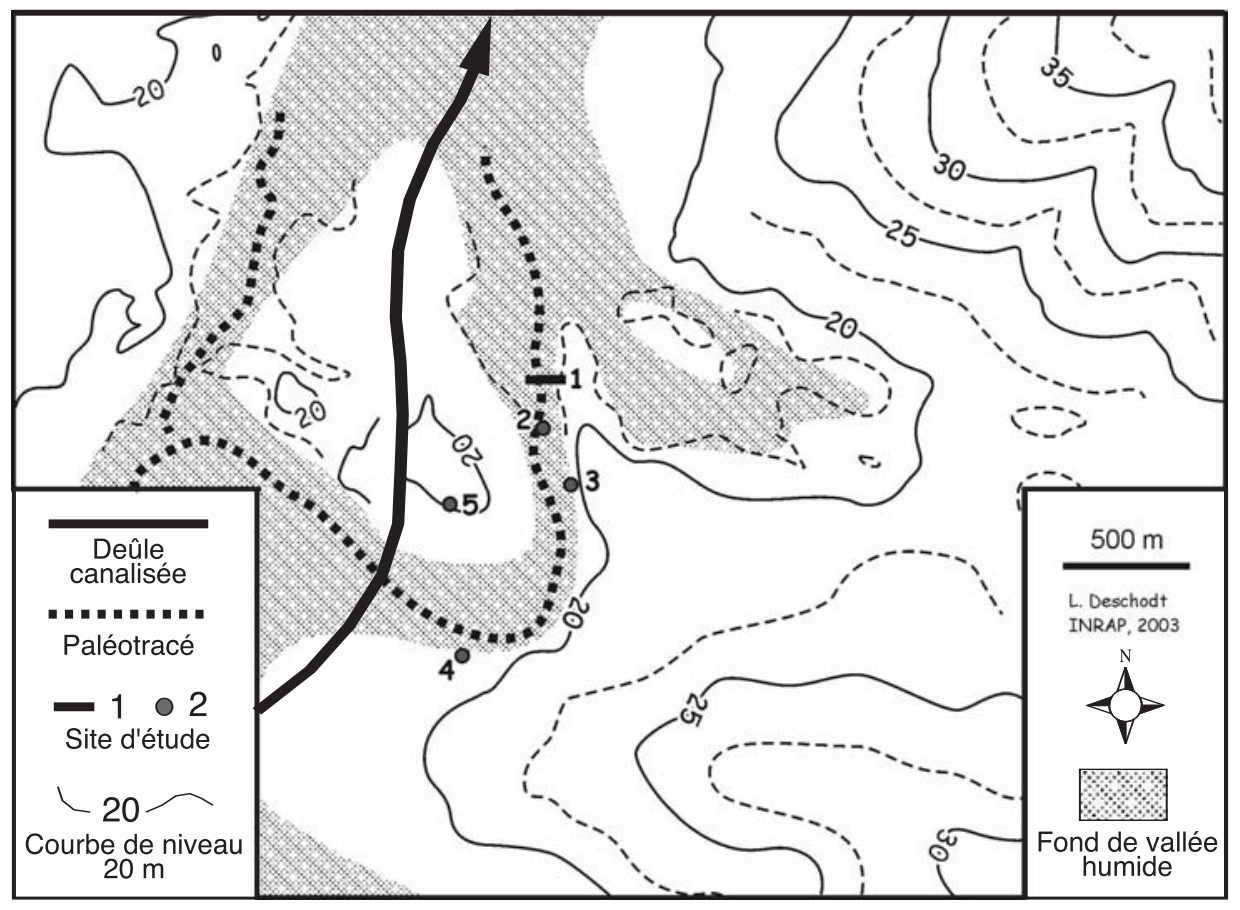

FIG. 3. - Localisation des sites d'investigation dans leur cadre topographique.

La Deûle à Houplin-Ancoisne est caractéristique des rivières du bas pays: une large vallée pour un faible débit, un envahissement loessique, des pentes douces mais un relief de détail sensible, le tout déterminant un fort potentiel d'enregistrement sédimentaire. Et, à l'image des autres rivières du bas pays, la vallée a subi une forte anthropisation: depuis longtemps le canal s'est substitué à la rivière et tend à annihiler son souvenir. Les métamorphoses de la rivière et du fond de vallée, ainsi que les occupations humaines associées à ses différentes phases restent d'autant plus obscures. Seule une étude stratigraphique des alluvions peut permettre de retrouver la Deûle passée et contribuer à comprendre ses riverains et leur environnement.

\subsection{Une accumulation d'observations stratigra- phiques dans le secteur d'Houplin-Ancoisne}

\subsubsection{Premières observations dans le lit mineur sur le site de la « station d'épuration » en 1999}

En 1999, la mise aux normes européennes de la station d'épuration d'Houplin-Ancoisne a été précédée

19. - L. DeschodT et Y. CRÉTEUR, Houplin-Ancoisne, la station d'épuration, sondages, document final de synthèse, SRA Nord-Pas de Calais, INRAP. Rapport non publié, 1999, $17 \mathrm{p}$.

20. - Il s'agit des Cypéracées $(53,3 \%)$ et de l'ensemble des plantes aquatiques $(8,6 \%)$. Cependant, la ripisylve n'est pas enregistrée. La de sondages archéologiques en puits, à la pelle mécanique, sur l'emplacement d'un futur bâtiment $\left(\mathrm{n}^{\circ} 2\right.$, fig. 3). L'aire sondée, peu étendue, se situe au pied du versant exposé à l'ouest, dans l'ancien lit mineur holocène devenu une friche marécageuse. Une approche stratigraphique ${ }^{19}$ et palynologique (analyses de M. Boulen, INRAP) est proposée, sur la base d'une coupe synthétique (fig. 4).

Sur une nappe de graviers de craie (6), les alluvions à proximité de la rive sont constituées d'un limon argilo-sableux gris à gravier et malacofaune (5). Cette unité est difficilement attribuable à une chronozone particulière. Peu diversifiée, la végétation majoritairement représentée est strictement locale, liée aux conditions édaphiques du site ${ }^{20}$. Ultérieurement, des dépôts graveleux, surtout constitués de malacofaunes, avec du bois flotté se déposent à l'ouest de la zone (7) tandis qu'un limon tourbeux, avec malacofaunes éparses (4) occupe la partie est de la zone, à une cote légèrement plus haute. L'ensemble est scellé par une tourbe limoneuse peu épaisse (3) qui a subi une phase de sécheresse (réseau de fentes de rétraction). Les

strate arborée $(\mathrm{AP}=24,4 \%)$ est dominée par le Pin $(16 \%)$ suivie du Noisetier $(6,6 \%)$. On trouve également, sporadiquement, quelques grains de Bouleau, Tilleul et Orme. Le reste de la strate herbacée est représenté par les Poacées et la présence de quelques taxons autres, en quantités très faibles et sans écologie particulière (M. Boulen, INRAP). 


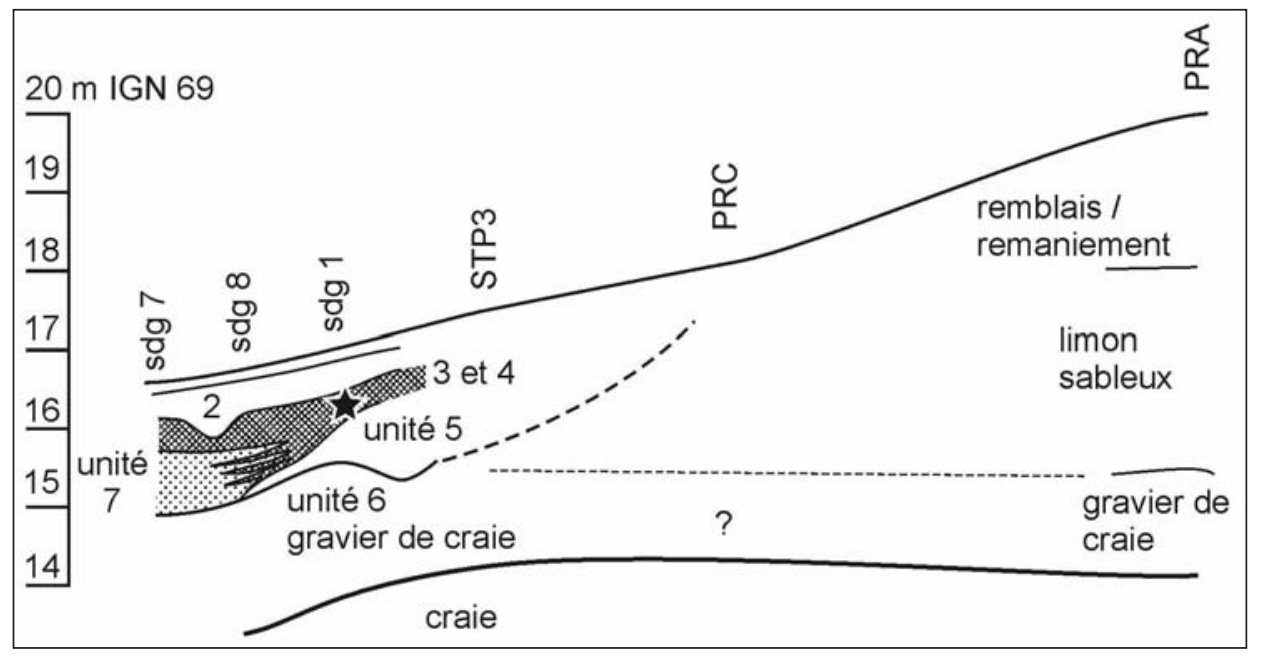

FIG. 4. - Coupe stratigraphique synthétique du site $n^{\circ} 2$ (station d'épuration, 1999).

spectres polliniques des unités tourbeuses (4 et 3 ) donnent l'image d'une végétation thermophile où la chênaie mixte est très bien représentée. La très grande extension du Tilleul, corrélée à un enregistrement faible de l'anthropisation, suggère une attribution à la palynozone Atlantique ${ }^{21}$. L'ensemble de la zone est par la suite colmaté par un limon argileux brun jaune (2) enrichi de grains de craie épars ou de fragments de briques à son sommet. Il s'agit d'un dépôt d'inondations historiques. Le sondage 1 a livré des ossements à $70 \mathrm{~cm}$ de profondeur, dans une petite fosse creusée dans les unités 3 et 4 . Il s'agit d'un dépôt de sept mâchoires inférieures de sangliers ou de porcs rustiques mâles avec usure prononcée des dents (description F. Decanter, INRAP). Une datation radiocarbone (GRA-19038 [Lyon-1547]) sur les ossements permet de les attribuer au Néolithique moyen: $5480+/-70$ BP, soit entre 4456 à 4115 av. J.-C. avec un intervalle de confiance de $95 \%$. Cette date est concordante avec la palynozone Atlantique proposée pour les unités tourbeuses.

\subsubsection{Des observations menées de part et d'autre du lit mineur entre 2001 et 2003}

Plusieurs opérations archéologiques ont permis de réaliser un levé des formations sédimentaires à proximité du lit mineur. En 2001, à proximité de la station d'épuration $\left(\mathrm{n}^{\circ} 3\right.$, fig. 3), la fouille programmée du

21. - Dans les unités 3 et 4, AP atteint des valeurs voisines de $80 \%$. Ce phénomène est imputable à la très grande représentation de la chênaie mixte à Tilleul (plus de $30 \%$ ), Chêne $(5,5$ et $2,4 \%)$, Orme $(1,4$ et $2,7 \%)$ et même Charme $(0,7 \%)$. La ripisylve est également bien représentée, essentiellement par l'Aulne (21,7 et 14,2\%). Le Noisetier est bien présent avec $9,5 \%$, ainsi que le Pin avec presque $15 \%$. La strate site laténien (G. Leman) et la fouille préventive du site néolithique (E. Martial, en cours d'étude) ont révélé la bonne conservation d'un sol tardiglaciaire-holocène développé sur un loess. Ce dernier surmonte des formations fluviatiles antérieures. Le sol a été préservé par des colluvions où se sont développés des horizons humifères datés du Néolithique et de l'âge du fer (G. Leman et al., ici même).

En 2002 et 2003, les opérations de fouilles préventives du « Marais de Santes / Jardin des communautés » (I. Praud, en cours d'étude, $n^{\circ} 4$, fig. 3 ) et les sondages rue G. Mocquet (E. Marial, $n^{\circ} 5$, fig. 3) ont donné lieu à un levé de détail du contact entre les formations loessiques et les formations fluviatiles sableuses antérieures (fig. 5). Ces relevés démontrent l'extension considérable des formations alluviales pléistocènes scellées par des formations loessiques « de fond de vallée », souvent litées, dans des conditions topographiques différentes.

\subsection{Un transect partiel de la vallée de la Deûle à Houplin-Ancoisne en 2003}

\subsubsection{Intérêt de la démarche}

Les données recueillies lors des opérations archéologiques sont dispersées et ne suffisent pas à comprendre le fonctionnement hydrologique global de la Deûle au cours du temps. Ces données sont pourtant

herbacée est relativement peu diversifiée. Elle est composée de Cypéracées, de Poacées, de quelques aquatiques et autres taxons non significatifs. Les indices d'anthropisation y sont quasiment absents à l'exception notable de deux grains de pollen de céréales (unité 3) (M. Boulen, INRAP). 


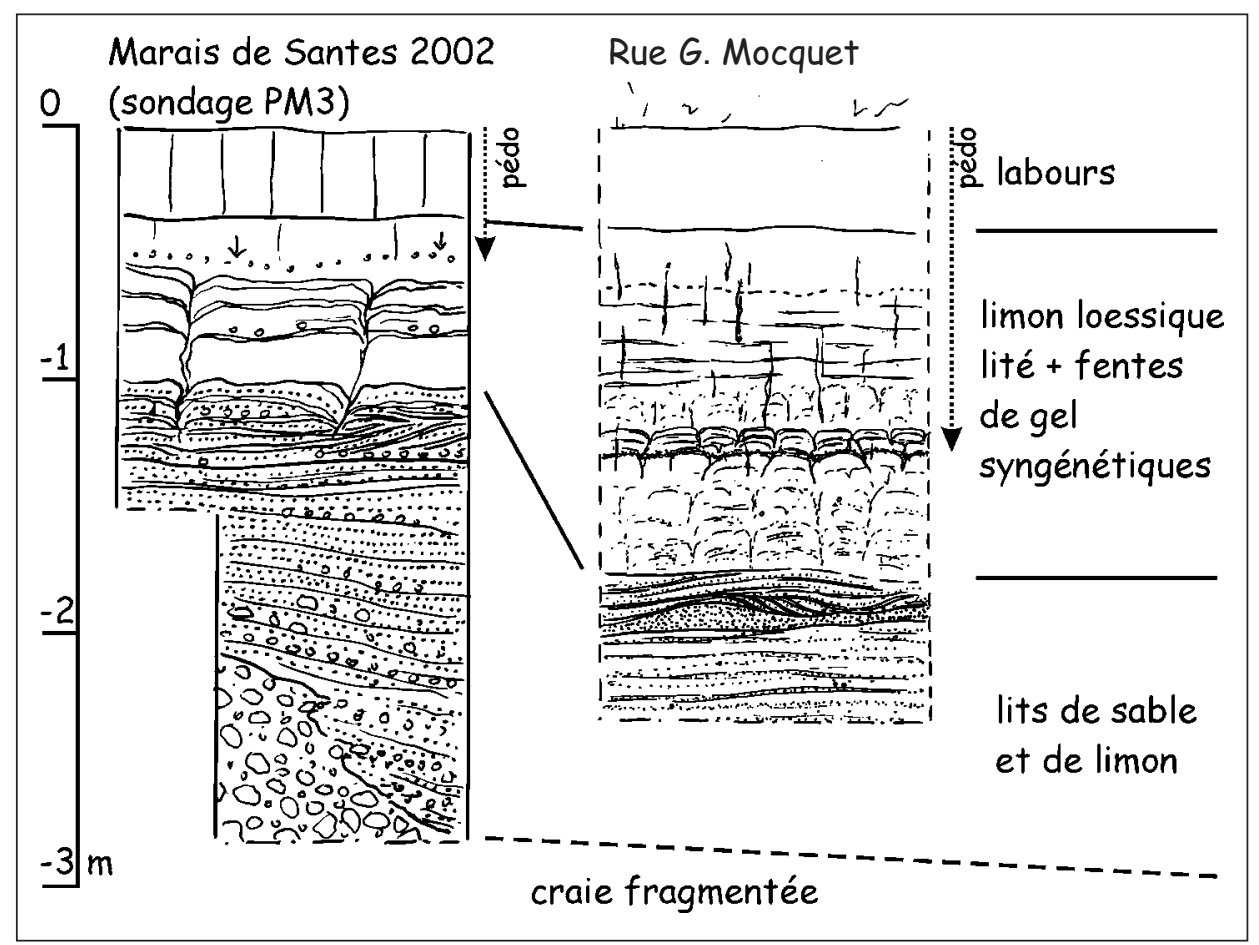

FIG. 5. - Logs stratigraphiques des sites $n^{\circ} 4$ (Marais de Santes) et $n^{\circ} 5$ (rue G. Mocquet).

essentielles pour comprendre la logique d'implantation des sociétés humaines. Les informations environnementales contemporaines des sites sont à rechercher dans les zones humides où sont susceptibles d'être conservés les meilleurs enregistrements sédimentaires. Nous avons donc entrepris la réalisation d'un transect de la vallée à la tarière à main $\left(\mathrm{n}^{\circ} 1\right.$ fig. 3 et fig. 6).

\subsubsection{Description du transect}

Les unités sédimentaires peuvent être décrites et synthétisées comme suit:

1. Craie: une craie massive, probablement la craie fragmentée en place, a été repérée dans le sondage S2 et le sondage S11 s'est arrêté sur cette même unité. Elle semble présenter un léger pendage vers l'est.

2. Craie fragmentée à matrice limoneuse: la craie en place est surmontée d'une couche de fragments de craie anguleux, de plusieurs centimètres, pris dans une pâte crayeuse.

3. Formations fluviatiles antérieures au dépôt de loess: tous les sondages ont rencontré des formations alluviales anciennes. À l'ouest il s'agit de lits de graviers de craie roulés, sans matrice ou avec un peu de sable, recouverts de fins lits de sable et de limon. Vers l'est, les faciès sont beaucoup plus diversifiés - lits de sable, de graviers de craie, passages limoneux - à tel point qu'il n'est pas possible d'établir de corrélations entre sondages. Un fragment de bois a été prélevé dans S5 («ij », fig. 6) et a donné une datation de $4135 \pm 40$ années BP (GrA-23285), rejetée en raison de son incohérence chronologique au sein du dépôt (racine ou élément retombé dans le sondage). Des dépôts tourbeux avec malacofaunes sont également repérés à $2 \mathrm{~m}$ de profondeur dans le sondage S2.

4. Loess: les formations alluviales sont recouvertes par un limon loessique homogène, supportant un sol brun sous forêt (S8) ou un champ labouré (S7) en pente douce (versant).

5. Formations fluviatiles tardiglaciaires: un chenal incise nettement les dépôts graveleux. La base du remplissage S11 est composée d'environ $40 \mathrm{~cm}$ d'un limon plastique clair coquillier dans lequel s'intercalent quelques fines lamines sableuses ou enrichies de très petits charbons de bois. Un de ceux-ci ( « a ») a donné un âge de $12560 \pm 100$ années BP, soit une attribution au Bølling (premier épisode chaud du Tardiglaciaire weichsélien).

Le remplissage de base du chenal est recouvert sur $45 \mathrm{~cm}$ d'épaisseur par un limon brun organique lité, 


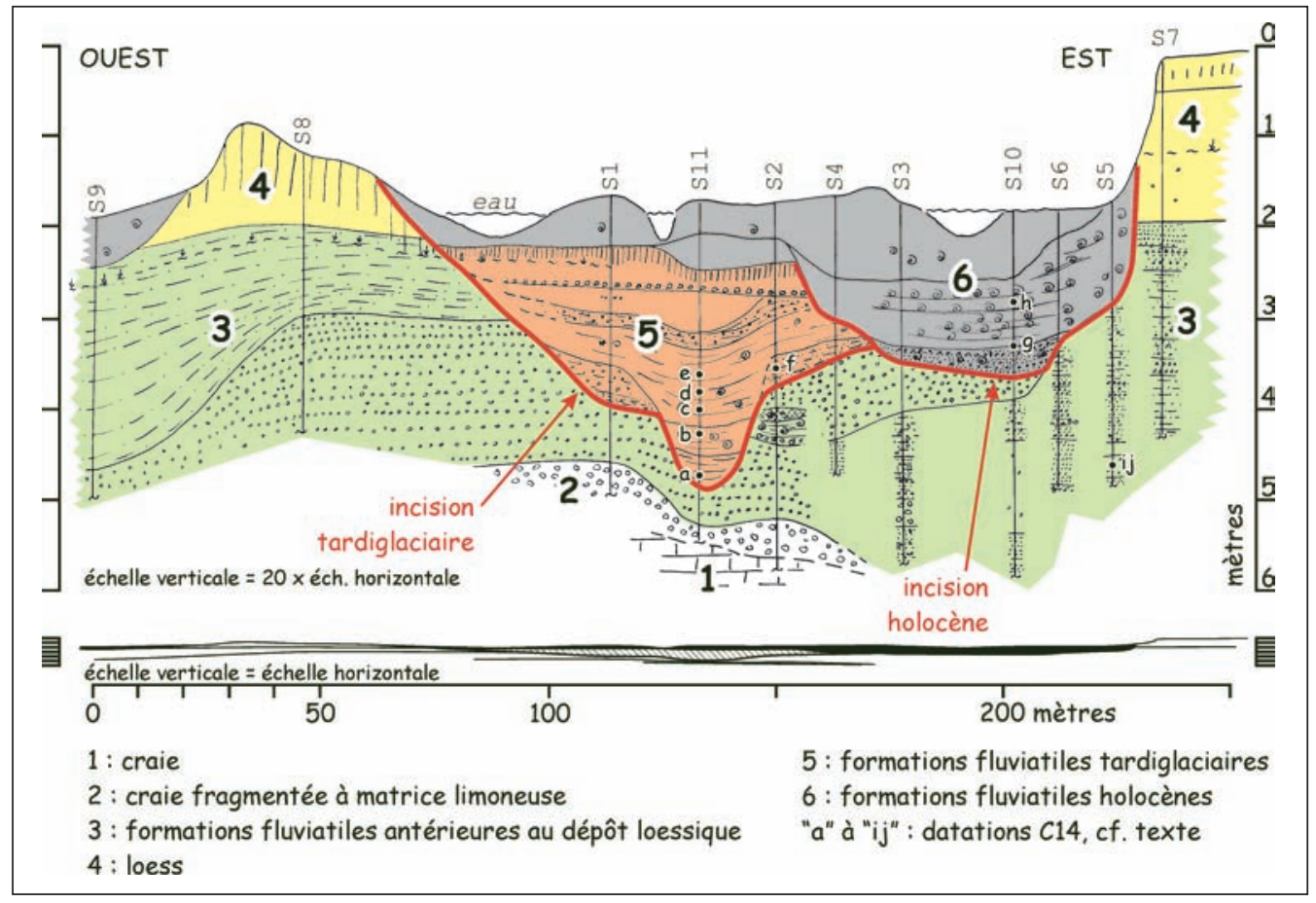

FIG. 6. - Coupe stratigraphique recoupant le paléotracé de la Deûle (point $n^{\circ} 1$ sur la fig. 3).

avec malacofaunes abondantes, bois et charbon de bois. Un petit bois flotté a donné une date de 10080 $\pm 60 \mathrm{BP}(\mathrm{GrA}-23291)$. On observe ensuite $90 \mathrm{~cm}$ de sédiment lité, limoneux et sableux, avec petits graviers, lits de sables et malacofaunes. Des lamines plus organiques ont donné trois dates homogènes: « $\mathrm{c}$ » $10410 \pm 60 \mathrm{BP}(\mathrm{GrA}-23290)$, « $\mathrm{d} » 10530 \pm 60 \mathrm{BP}(\mathrm{GrA}-23289)$ et $« \mathrm{e} » 10570 \pm$ 60 (GrA-23287). Cet ensemble de dates place sans ambiguïté ces unités et la fin du remplissage du chenal au cours du Dryas récent. De même, latéralement, les faciès légèrement différents - gravier de craie à matrice limonseuse (S1) ou sable limoneux lité (S2) - sont attribuables au même épisode froid («f » $10190 \pm 60 \mathrm{BP}$ ). La fin du remplissage du chenal tardiglaciaire est composé d'une unité sablo-graveleuse et de deux limons gris homogènes séparés par un niveau érosif à graviers de craie anguleux. Un petit sol s'est développé au sommet de la série.

6. Formations fluviatiles holocènes: le chenal holocène ne se surimpose pas au chenal tardiglaciaire. Il est décalé vers l'est et entaille les formations antérieures. Le bras est colmaté à la base par un sable lité graveleux, à gravier de craie et malacofaunes brisées (environ $30 \mathrm{~cm}$ d'épaisseur). Puis un limon tourbeux lité, à nombreuses malacofaunes remplit la majeure partie du lit mineur. Vers le centre de celui-ci (S10), le sédiment est presque parfois exclusivement composé d'un amas de coquilles. Deux datations permettent d'associer ce dépôt à un épisode relativement long allant du Boréal au Subboréal: la transition entre sable et limon tourbeux ( g ») est datée de $8380 \pm 50$ années BP (GrA-23190) ; et, au sein de l'unité, un bois flotté (« $\mathrm{h} »)$ donne un âge de $3590 \pm 40$ années BP (GrA-23286), soit entre 2107 et 1780 avant J.-C. avec une probabilité de $95 \%$. La fin du comblement se compose d'un limon brun, proche du précédent, mais au caractère tourbeux un peu moins prononcé.

\subsubsection{Interprétation des données stratigraphiques}

\section{Les nappes fluviatiles anciennes}

Le transect partiel réalisé en 2002 à HouplinAncoisne illustre remarquablement le potentiel d'enregistrement et la complexité des alluvions pléistocènes dans la vallée de la Deûle. Les dépôts loessiques (4) scellent des nappes fluviatiles anciennes emboîtées (3). Le contact entre la craie en place et les nappes fluviatiles présente un léger pendage vers l'est, opposé au versant. Le fait est d'ailleurs sensible à travers les sondages géotechniques de la station d'épuration (fig. 4). L'extension 
précise des nappes pléistocènes n'est pas encore connue. Elles s'étendent probablement à la majeure partie du fond de vallée actuel, voire au-delà, et recèlent un potentiel archéologique évident.

\section{Le limon loessique de fond de vallée}

Le dépôt loessique (4) est continu dans le fond de vallée quand il n'a pas été incisé ou recouvert par des sédiments fluviatiles postérieurs. Les observations directes (fig. 5) de la partie non atteinte par la pédogenèse montrent que, malgré son origine éolienne, il présente un faciès de remaniement fluviatile (litages, éventuellement lits de sable ou de graviers de craie). La présence fréquente de petites fentes de dessiccation syngénétiques (développées pendant le dépôt de l'unité) témoigne de la rapidité de la sédimentation pendant laquelle se sont succédées de nombreuses phases sèches. Ce faciès « de fond de vallée » est interprété comme un remaniement du loess par des écoulements en nappe lors du dégel saisonnier ${ }^{22}$. Il passe latéralement à un faciès de loess typique sur versant, déposé lors du Pléniglaciaire weichsélien (maximum du froid du dernier glaciaire) $)^{23}$.

\section{Un enregistrement tardiglaciaire original (5)}

À la transition Pléniglaciaire-Tardiglaciaire, la reprise d'un écoulement concentré permet la création d'un chenal. La date réalisée à la base de son comblement est une des plus anciennes obtenue pour le Bølling (vers 12500 BP contre environ 12400-12300 $\mathrm{BP}$ à la base des chenaux dans le Bassin Parisien $)^{24}$. Cette précocité témoigne peut-être de la rapidité de la réponse morphologique à un changement climatique dans le petit bassin versant de la Deûle.

Sans que nous puissions distinguer de périodes intermédiaires (Allerød...), la majeure partie du comblement est datée de la fin du Tardiglaciaire et plus précisément du Dryas récent. Un problème de datation peut éventuellement se poser pour la datation $\langle\mathrm{b} »$, légèrement plus récente que les dates positionnées au-dessus. Elle correspond par ailleurs à un faciès tourbeux a priori peu compatible avec la péjoration climatique froide. En tout cas, les dates suivantes $(\mathrm{c}, \mathrm{d}, \mathrm{e})$ sont homogènes et associées à un remplissage lité à macrorestes végétaux. Ce faciès de

22. - L. DESCHODT, Ébauche d'une cartographie..., op. cit. 23. - J. Sommé, Les plaines du Nord de la France..., op. cit.; P. Antoine, P. Auguste, J.-J. Bahain, P. Coudret, P. Depaepe, J.-P. Fagnart, C. Falguères, M. Fontugne, M. Frechen, C. Hatté, A. Lamotte, M. Laurent, N. Limondin-Lozouet, J.-L. Locht, N. Mercier, A.-M. Moigne, A.-V. Munaut, P. Ponel, D.D. Rousseau, «Paléoenvironnements pléistocènes et peuplements paléolithiques dans la Somme (Nord de la France) », Bulletin de la Société préhistorique française, 2003, t. 100, n 1 , p. 5-28.

24. - J.-F. Pastre, C. Leroyer, N. Limondin-Lozouet, C. Chausse, chenal Dryas récent est ici extrêmement bien représenté alors que dans les grandes vallées du Bassin Parisien, il est difficile à détecter au sein d'un faciès homogène de dépôts d'inondation ${ }^{25}$.

Nous ne possédons pas de datation radiocarbone pour la suite du comblement. Sa stratigraphie est d'une complexité inédite pour le Dryas récent: un niveau détritique grossier, un limon homogène, une limite érosive nette avant un nouveau dépôt limoneux homogène. Toutefois, la géométrie du dépôt détritique, qui l'inclut d'emblée dans le comblement du chenal tardiglaciaire, l'aspect azoïque de l'ensemble et l'absence d'une quelconque décarbonatation avant le petit sol sommital plaident pour une attribution tardiglaciaire.

\section{L'enregistrement holocène (6)}

À la transition Tardiglaciaire-Holocène, vers 10000 $\mathrm{BP}$, le retour à un climat tempéré et humide favorise une nouvelle incision du lit mineur. Ce dernier migre vers l'est et épargne le chenal tardiglaciaire. Le déplacement du cours a peut-être été préparé par les conditions micro-topographiques de la toute fin du Dryas récent (léger pendage des unités supérieures vers l'est). Formée dans les formations pléistocènes sableuses et loessiques, la berge est marquée et conserve encore aujourd'hui vigueur et fraîcheur. Elle définit une terrasse alluviale qui, au début de l'Holocène, dominait une terrasse plus basse de $1 \mathrm{~m}$ comportant un petit sol et dont les berges s'ouvraient dans les alluvions tardiglaciaires. Ces unités de terrain, différenciées non seulement par leur morphologie mais aussi par la proximité de la nappe, la nature du sédiment, leur inondabilité, et donc par la nature du couvert végétal, s'étiraient le long du lit de la Deûle. Elles offraient à l'homme des conditions d'implantation et d'exploitation du milieu diversifiées, et impliquent aujourd'hui autant de logiques taphonomiques pour l'archéologue.

Dès le Boréal (datation «g $»$ ), la dynamique du cours d'eau diminue et le lit est envahi par un sédiment tourbeux. La charge détritique est extrêmement faible et une part importante du comblement est le fait de mollusques aquatiques. Une corrélation évidente

M. Fontugne, A. Gebhardt, C. Hatte, V. Krier, « Le Tardiglaciaire des fonds de vallée du Bassin Parisien (France) », Quaternaire, 11, (2), 2000, p. 107-122; P. ANTOINE, J.-P. FAGNART, N. Limondin-LOZOUET, A.-V. Munaut, « Le Tardiglaciaire du Bassin de la Somme: éléments de synthèse et nouvelles données », Quaternaire, vol. 11, n² 2, 2000, p. 85-98.

25. - P. AnToINE, « Évolution Tardiglaciaire et début Holocène des vallées de la France septentrionale: nouveaux résultats », Comptes Rendus de l'Académie des Sciences de Paris, Sciences de la terre et des planètes, $1997, \mathrm{n}^{\circ} 325$, p. 35-42. 
avec les unités 3, 4 et 7 de l'observation de 1999 peut être faite (fig. 4). La palynozone Atlantique proposée pour le développement tourbeux sur le site de la station d'épuration est encore une fois cohérente avec les datations radiocarbones puisque le même processus de dépôt en eau calme se poursuit encore sans rupture durant le Subboréal au droit du transect (datation « $\mathrm{h} »)$. L'atterrissement de la partie orientale du cours était plus avancée en amont (sous la station d'épuration) puisqu'un Néolithique y a creusé une fosse.

Le comblement s'est poursuivi jusqu'à envahir la berge occidentale du cours d'eau et transformer en marécage de vastes étendues situées entre les berges et les « îlots » loessiques, aboutissant à l'état actuel du fond de vallée.

\section{CONCLUSION: L'AMORCE D'UNE RÉFLEXION PALÉOENVIRONNEMENTALE À L'ÉCHELLE DE LA VALLÉE?}

Ce transect partiel, accompagné de datations radiocarbones, apporte des données stratigraphiques à la compréhension du paléoenvironnement de la Deûle. Toutefois l'information est incomplète. L'étude porte sur une partie du fond de vallée et ne permet pas d'observer des phases d'évolution complémentaires pouvant se dérouler simultanément à quelques centaines de mètres plus à l'ouest (deux cours sont perceptibles sur les cartes topographiques). Par ailleurs, l'analyse est encore fragmentaire, faute d'avoir étudié les marqueurs biologiques ou lithologiques.

Toutefois, malgré les limites de la démarche, ce travail éclaire les conditions locales d'implantation des sites proches: localisation sur une berge marquée offrant un accès facile au lit mineur, atterrissement progressif de ce dernier et variation de la largeur de la zone marécageuse au cours de l'Holocène, présence d' « îlot» au sein de la zone humide. À l'échelle de la vallée, l'analyse stratigraphique contribue aux problématiques géoarchéologiques: pourquoi, par exemple, observe-t-on une reprise de l'érosion durant l'époque gallo-romaine à Lille ${ }^{26}$, alors qu'elle n'est pas perçue dans le lit mineur à Houplin-Ancoisne? Est-ce une réponse à une évolution différenciée des activités humaines ou de la dynamique de la rivière?

En l'absence de cette approche pluridisciplinaire et globale, l'archéologue se priverait de la compréhension du milieu (hydrologie, évolution du cours d'eau et de sa vallée, végétation...) mais également de la perception des liens qu'entretenaient les hommes avec leur environnement immédiat (choix d'implantation, d'exploitation des ressources...).

Mots-clés : Deûle, fond de vallée, paléoenvironnement, Tardiglaciaire, Holocène, dynamique fluviale. 